\title{
Gerenciamento de resíduos de saúde no Brasil: Desafios de gestores e profissionais de
}

\section{saúde}

\author{
Health management in Brazil: Challenges of managers and health professionals waste \\ Gestión de residuos sanitarios en Brasil: Retos de gestores y profesionales de la salud
}

Recebido: 16/03/2021 | Revisado: 22/03/2021 | Aceito: 26/03/2021 | Publicado: 03/04/2021

\author{
Raquel Maria de Sena \\ ORCID: https://orcid.org/0000-0001-9913-5076 \\ Universidade Federal Fluminense, Brasil \\ E-mail: raquelrq11@hotmail.com \\ Maritza Consuelo Ortiz Sanchez \\ ORCID https://orcid.org/0000-0003-0131-9489 \\ Universidade Federal Fluminense, Brasil \\ E-mail: morsa_peru@yahoo.com \\ Érica Brandão de Moraes \\ ORCID: https://orcid.org/0000-0003-3052-158X \\ Universidade Federal Fluminense, Brasil \\ E-mail: enfermeira_erica@yahoo.com.br \\ Maria Lelita Xavier \\ ORCID: https://orcid.org/0000-0003-3014-733X \\ Universidade Federal Fluminense, Brasil \\ E-mail: lely108@hotmail.com \\ Andre Luiz de Souza Braga \\ ORCID: https://orcid.org/0000-0002-7961-9038 \\ Universidade Federal Fluminense, Brasil \\ E-mail: andre.braga @ globo.com \\ Mônica Aparecida de Oliveira Pinto Porto \\ ORCID: https://orcid.org/0000-0001-5904-0320 \\ Universidade Federal Fluminense, Brasil \\ E-mail: enfmonicaporto@gmail.com
}

\begin{abstract}
Resumo
Objetivo: analisar nas produções científicas as evidências relacionadas aos desafios da equipe multidisciplinar no gerenciamento de resíduos de saúde na prevenção e controle de infecção hospitalar. Método: Quais os desafios da equipe multidisciplinar no gerenciamento de resíduos de saúde visando a prevenção e controle de infecção hospitalar? Revisão Integrativa de abordagem qualitativa, com busca de artigos nas bases de dados que compõem a Biblioteca Virtual em Saúde. Resultados: 10 artigos foram incluídos, para a construção desta revisão integrativa e ordenados de forma a atingir o objetivo da pesquisa. Conclusão: As evidências mostram que a equipe multidisciplinar enfrenta desafios estruturais e de adesão ao plano de gerenciamento de resíduos. No entanto, vale ressaltar que tanto a educação permanente voltada para a capacitação dos funcionários e gestores quanto ao gerenciamento dos RSS apropriado, contribuem no controle de infecção garantindo a segurança e reduzindo as ameaças de exposição à saúde humana.
\end{abstract}

Palavras-chave: Pessoal da saúde; Gerenciamento de resíduos; Resíduos de serviços de saúde; Controle de infecções; Infecção hospitalar.

\begin{abstract}
Objective: to analyze in scientific productions the evidence related to the challenges of the multidisciplinary team in the management of health waste in the prevention and control of hospital infection. Method: What are the challenges of the multidisciplinary team in the management of health waste aiming at the prevention and control of hospital infection? Integrative Review of qualitative approach, searching for articles in the databases that make up the Virtual Health Library. Results: 10 articles were included, for the construction of this integrative review and ordered by didactic similarity according to the challenges of the multidisciplinary team. Conclusion: Evidence shows that the multidisciplinary team faces structural and compliance challenges in waste management. However, it is worth mentioning that both permanent education aimed at training employees and managers and the management of appropriate RSS, contribute to infection control, ensuring safety and reducing threats of exposure to human health.

Keywords: Health personnel; Waste management; Medical waste; Infection control; Cross infection.
\end{abstract}




\section{Resumen}

Objetivo: analizar en producciones científicas las evidencias relacionadas con los retos del equipo multidisciplinario en la gestión de residuos sanitarios en la prevención y control de la infección hospitalaria. Método: ¿Cuáles son los retos del equipo multidisciplinario en la gestión de residuos sanitarios con miras a la prevención y control de la infección hospitalaria? Revisión integradora del enfoque cualitativo, búsque da de artículos en las bases de datos que componen la Biblioteca Virtual en Salud. Resultados: Se incluyeron 10 artículos, para la construcción de esta revisión integradora y ordenados por similitud didáctica de acuerdo a los desafíos del equipo multidisciplinario. Conclusión: La evidencia muestra que el equipo multidisciplinario enfrenta desafíos estructurales y de cumplimiento en la gestión de residuos. Sin embargo, cabe mencionar que tanto la educación permanente dirigida a la formación de empleados y directivos como el manejo de RSS adecuados, contribuyen al control de infecciones, garantizando la seguridad y reduciendo las amenazas de exposición a la salud humana.

Palabras clave: Personal de salud; Administración de residuos; Residuos sanitarios; Control de infecciones; Infección hospitalaria.

\section{Introdução}

Tanto a Resolução da Diretoria Colegiada (RDC) nº 222/18 da Agência Nacional de Vigilância Sanitária (ANVISA) quanto a RDC no 358/2005 do Conselho Nacional de Meio Ambiente (CONAMA) conceituam Resíduos Sólidos de Saúde como todo resíduo produzido no decurso das atividades desempenhadas através dos geradores dos resíduos de serviços de saúde humana ou animal, sendo divididos em cinco grupos: grupo A - biológicos, grupos B - químicos, grupo C - radioativos, grupo D - comuns e grupo E - perfuro cortantes (Ministério da Saúde, 2018; Conselho Nacional do Meio Ambiente, 2005).

Cabe ressaltar que a Organização Mundial da Saúde (OMS) destaca que os percentuais de resíduos produzidos durante as atividades na assistência à saúde, giram em torno de $85 \%$ de resíduos gerais e não perigosos, em comparação aos resíduos domésticos. E 15\% são caracterizados como materiais perigosos, podendo ser infecciosos, químicos ou radioativos (World Health Organization, 2018).

Observa-se ainda que no mundo, cerca de 16 bilhões de injeções são administradas anualmente, mas não são todas as agulhas e seringas que são desprezadas de forma adequada, assim como luvas, frascos e ampolas, potencializando o risco de ferimentos, infecções e favorecendo circunstância para a reutilização (World Health Organization, 2018).

Os resíduos produzidos pelos serviços de saúde podem ser perigosos, tóxicos e letais por possuírem um grande potencial de infecção, de transmissão de doenças, os quais podem gerar graves consequências tanto para o meio ambiente quanto para a população. Isto, já está comprovado e desta forma merece assim, um destaque nas discussões, pelos impactos que podem ocasionar (Sodré \& Lemos, 2017).

A alta produção de resíduos sólidos provocou inúmeros problemas durante anos, quanto ao seu descarte ideal, despertando no poder público a preocupação com a implementação de providências para o correto descarte dos mesmos, visando a possibilidade de diminuição dos impactos ambientais (Silva, 2019).

Embora nos últimos anos tenha sido demostrado uma redução das injeções com agulhas e seringas contaminadas nos países de baixo rendimento, a OMS destaca que em 2010, as injeções com descarte inadequadas ainda foram responsáveis por 33.800 novas infecções por HIV, 1,7 milhão de infecções por hepatite B e 315.000 infecções por hepatite C (World Health Organization, 2018).

Estima-se que um profissional apresenta riscos de 30\%, 1,8\% e 0,3\% respectivamente, de se infectar com Hepatite B (HBV), Hepatite C (HCV) e Imunodeficiência Humana (HIV), ao sofrer uma lesão por picada de agulha usada de um paciente fonte (World Health Organization, 2018)

Em 2015, a Organização Mundial da Saúde (OMS) e Fundo das Nações Unidas para a Infância (UNICE) avaliaram de forma conjunta e constataram que 58\% das instalações existentes de 24 países possuem sistemas adequados para o descarte de resíduos de serviços de saúde (World Health Organization, 2018).

O Brasil retrata uma preocupação com os resíduos sólidos que são produzidos nos serviços de saúde. Esta 
preocupação não se deve pelo volume gerado, mas sim, pelo alto potencial de risco que pode refletir na comunidade e no meio ambiente, pois são fontes de organismos patogênicos, produtos tóxicos, inflamáveis, perfurocortantes e radioativos favorecendo a contaminação ambiental, incluindo risco adicional aos profissionais envolvidos no seu manejo e a comunidade geral, conforme mencionado linhas acima (Sodré \& Lemos, 2017; Ministério da Saúde, 2006).

Em questão aos resíduos de Serviços de Saúde (RSS), é relevante destacar que das 149.000 toneladas de resíduos residenciais e comerciais geradas diariamente, apenas uma fração inferior a $2 \%$ é composta por RSS e, destes, apenas 10 a $25 \%$ necessitam de cuidados especiais. À vista disso, a implantação de processos de segregação dos diferentes tipos de resíduos em sua fonte e no momento de sua geração conduz certamente à minimização de resíduos, em especial àqueles que necessitam de um tratamento prévio à disposição final (Ministério da Saúde, 2006).

De forma global, nos últimos anos aumentou a preocupação sobre a questão da gestão dos RSS, no que se refere ao seu gerenciamento seguro e adequado, em virtude de terem a consciência nesses últimos dez anos dos riscos impostos à saúde pública e ambiental, de uma gestão inadequada dos RSS (Sodré \& Lemos, 2017).

Desta forma, a RDC 222/18 determina que os prestadores de serviços que geram resíduos de serviços de saúde devem possuir um Plano de gerenciamento de RSS (PGRSS), o qual deve ser elaborado com base nas peculiaridades de cada serviço gerador de RSS bem como na sua demanda. E com observância das regulamentações federais, estaduais, municipais ou Distrito Federal (Ministério da Saúde, 2018; Conselho Nacional do Meio Ambiente, 2005).

Com esse entendimento a equipe multidisciplinar, tem papel fundamental no gerenciamento dos resíduos gerados pelos serviços de saúde; para isto devem-se considerar as diversas etapas: geração, segregação, acondicionamento, transporte, até a disposição final, proporcionando que se definam de forma sistemática e integrada, em cada uma delas, metas, programas, sistemas organizacionais e tecnologias, compatíveis com a realidade local.

Além das práticas, os procedimentos técnicos, a recomendação dos descartes determinada pelo gerenciamento de resíduos de saúde acentua-se a prevenção e controle da infecção relacionada à assistência à saúde (IRA), que de acordo com a Portaria n. 2616/98 do Ministério da Saúde é aquela adquirida após admissão do paciente e que se manifeste durante a internação ou após a alta, quando relacionada com a internação ou a procedimentos hospitalares / ambulatoriais ou as manifestadas antes de 72 horas da internação, mas deve estar associada a procedimentos diagnósticos e/ ou terapêuticos, realizados durante este período (Ministério da Saúde, 1998). Essas associações são de extrema importância para o êxito das ações de controle de infecção, o envolvimento, a participação e a integração da Comissão de Controle de Infecção Hospitalar (CCIH) com os serviços de apoio do hospital (Alves \& Lacerda, 2015; Giarola et al., 2010).

Tendo em vista de que os resíduos produzidos nos serviços de saúde demonstram um grave problema, o qual potencializará o aumento da taxa de infecções relacionadas à assistência à saúde de forma geral, este estudo tem como objetivo analisar nas produções científicas as evidências relacionadas aos desafios da equipe multidisciplinar no gerenciamento de resíduos de saúde na prevenção e controle de infecção hospitalar.

\section{Metodologia}

A pesquisa é de abordagem qualitativa, entende-se que este tipo de abordagem torna-se importante a prática reflexiva de ênfase social que se investiga e do processo de investigação. Dessa forma, a pesquisa qualitativa desenvolve as percepções e interpretações subjetivas, que surgem da experiência; comportamentos objetivos, que surgem das ações; e o contexto, que envolve os aspectos sociais, culturais, políticos e físicos que rodeiam os sujeitos da pesquisa. (Pereira et al.,2018).

Este estudo configura-se como uma Revisão Integrativa (RI) da literatura, que se caracteriza por ser uma abordagem metodológica completa no que se dispõe às revisões, que permite uma percepção mais plena dos acontecimentos no âmbito da Prática Baseada em Evidências (PBE), visto que permite a inclusão de estudos de diferentes metodologias, além da 
combinação de literatura teórica e empírica, que se estendem desde a definição dos conceitos, revisão de teorias e evidencias até a análise concreta de dificuldades metodológicas de forma particular. Prática Baseada em Evidência (PBE) envolve a sistematização e publicação dos resultados de uma pesquisa bibliográfica em saúde para que possam ser úteis na assistência e gerenciamento à saúde, acentuando a importância da pesquisa acadêmica na prática clínica e gerencial (Tavares et al., 2010; Sasso et al., 2018).

Essa revisão seguiu as diretrizes PRISMA. Para a elaboração da revisão integrativa efetuou-se os seguintes passos: Elaboração da questão de pesquisa, busca na literatura; coleta de dados; análise crítica dos artigos; discussão dos resultados, apresentação da revisão e conclusão.

Estabeleceu-se na primeira etapa a questão norteadora com a seguinte indagação: Quais os desafios da equipe multidisciplinar no gerenciamento de resíduos de saúde visando a prevenção e controle de infecção hospitalar?

Para verificação na base Biblioteca Virtual em Saúde (BVS), foi aplicada a estratégia PICO e a utilização dos Descritores nas Ciências da Saúde da Biblioteca Virtual em Saúde (DeCS-BVS) para verificar qual descritor que se adéqua na estratégia PICO, em que a letra "P" representa paciente ou população, "I" refere-se à intervenção, "C" diz respeito à comparação com outros autores, que neste estudo, não se aplica, e a letra "O" nos remete aos resultados esperados.

O Quadro 1 descrito a seguir, expõe os descritores utilizados para filtragem dos artigos científicos.

Quadro 1- Descrição da estratégia PICO.

\begin{tabular}{|c|l|}
\hline Acrônimo & \multicolumn{1}{|c|}{ Descrição } \\
\hline P & Pessoal de saúde \\
\hline I & Gerenciamento de resíduos de saúde \\
\hline C & Não se aplica \\
\hline O & Prevenção e o controle da infecção hospitalar \\
\hline
\end{tabular}

Fonte: Dados inseridos na Biblioteca Virtual em Saúde (BVS), Base de dados MEDLINE, LILACS e BDENF.

A estratégia PICO norteia a elaboração da pergunta de pesquisa e do levantamento bibliográfico e possibilita o profissional da área assistencial e de pesquisa, localizar de forma consistente e fundamentada a informação científica mais adequada disponível, durante uma dúvida ou questionamento (Santos, 2007).

Foram utilizados para a seleção das produções os seguintes descritores: Pessoal da saúde (Health Personnel) AND Gerenciamento de resíduos (Waste Management) OR resíduos de serviços de saúde (health residues) AND Prevenção e controle OR Infecção hospitalar OR prevenção de saúde (Infection Control) OR (Control, Infection). Não foi utilizado a terminologia "Equipe multidisciplinar" devido não ser reconhecida pelo DECs, com isto utilizou-se o termo "Pessoal de saúde".

$\mathrm{Na}$ etapa seguinte, o portal da BVS remete as principais bases de dados, sendo selecionadas as seguintes: Bases da Literatura Latino-Americana e do caribe em Ciências da Saúde (LILACS), Bases de Dados de Enfermagem (BDENF) e (MEDLINE). Foram incluídas as produções científicas publicadas no período de 2015 a 2020, nos idiomas português, inglês ou espanhol, que abordavam o gerenciamento de resíduos de saúde na prevenção e controle da infecção hospitalar e no setor de gerenciamento de resíduos de saúde propriamente dito.

Para exprimir as informações dos estudos selecionados de forma organizada, e posteriormente a exibição dos resultados, elaborou-se instrumento que compreende os dados de identificação dos artigos tais como: autor, ano, título, país de publicação, periódico, área de conhecimento do primeiro autor, idioma da publicação, delineamento, principais resultados e resposta à questão da pesquisa quanto aos desafios para equipe multidisciplinar no que diz respeito ao gerenciamento de resíduos de saúde na prevenção e controle da infecção hospitalar descrita pelos autores. Os artigos extraídos estão em ordem de acordo com a letra inicial das bases de dados utilizadas, seguida do número conforme descrito nas referências bibliográficas. 
Sendo (M) para MEDLINE, LILACS descrito como (L) e BDENF exposto como (B). O instrumento também teve o intuito de sinalizar os dados pertinentes das amostras para que objetivo traçado fosse alcançado.

Os estudos foram agrupados por características de semelhanças didáticas quanto aos desafios da equipe multidisciplinar no que se refere ao gerenciamento de resíduos de saúde na prevenção e controle das IRAs (infecção relacionada a assistência a saúde). Com ênfase no delineamento dos estudos e com base nas características das mesmas, os artigos foram hierarquizados de acordo com cinco níveis de evidências (NE) que seguiram a Oxford Centre for Evidence Based-Medicine: 1A-Revisões sistemáticas e meta análises de ensaios clínicos comparáveis. Estudos controlados randomizados bem delineados com desfecho clínico relevante. 1B-Estudos controlados randomizados com estreito intervalo de confiança. 1C- Resultados do tipo "tudo ou nada". Estudo de série de casos controlados. 2A- Revisão sistemática homogênea de estudos de coorte (com grupos de comparação e controle de variáveis). 2B- Estudo de coorte com pobre qualidade de randomização, controle ou sem acompanhamento longo, estudo de coorte transversal. 2C- Resultados de pesquisas (observação de resultados terapêuticos ou evolução clínica). 3A- Revisão sistemática homogênea de estudos de caso com grupo-controle. 3B Estudos de caso com grupo-controle. 4- Relatos de caso e série sem definição de caso controle. 5- Opinião de autoridades respeitadas ou especialistas. Revisão da literatura não sistemática (Pedrosa et al., 2015).

$\mathrm{Na}$ análise realizada nos possibilitou visualizar duas categorias: desafios estruturais e desafios para adesão dos profissionais aos procedimentos.

\section{Resultados}

A busca pelas produções se deu até o dia quinze de maio de 2020 e resultou num total de 56 artigos publicados. Desses, 25 contidos na base de dados da MEDLINE, 16 LILACS e 15 BDENF. Dentre estes foram excluídas 16 publicações por duplicidade; 10 publicações foram excluídas por não atender o critério de inclusão. Após essas exclusões, foi realizada a leitura dos títulos e resumos das publicações, com o propósito de analisar a relevância do estudo para sua inclusão na amostra. Em seguida, excluíram-se 20 artigos por não responderem à questão da pesquisa. Dessa forma, 10 artigos foram incluídos, sendo 6 artigos da base de dados da LILACS, 3 na BDENF e 1 na MEDLINE para a construção desta revisão integrativa (Figura 1). 
Figura 1 - Fluxograma de seleção dos artigos analisados. Niterói (RJ), Brasil, 2020.
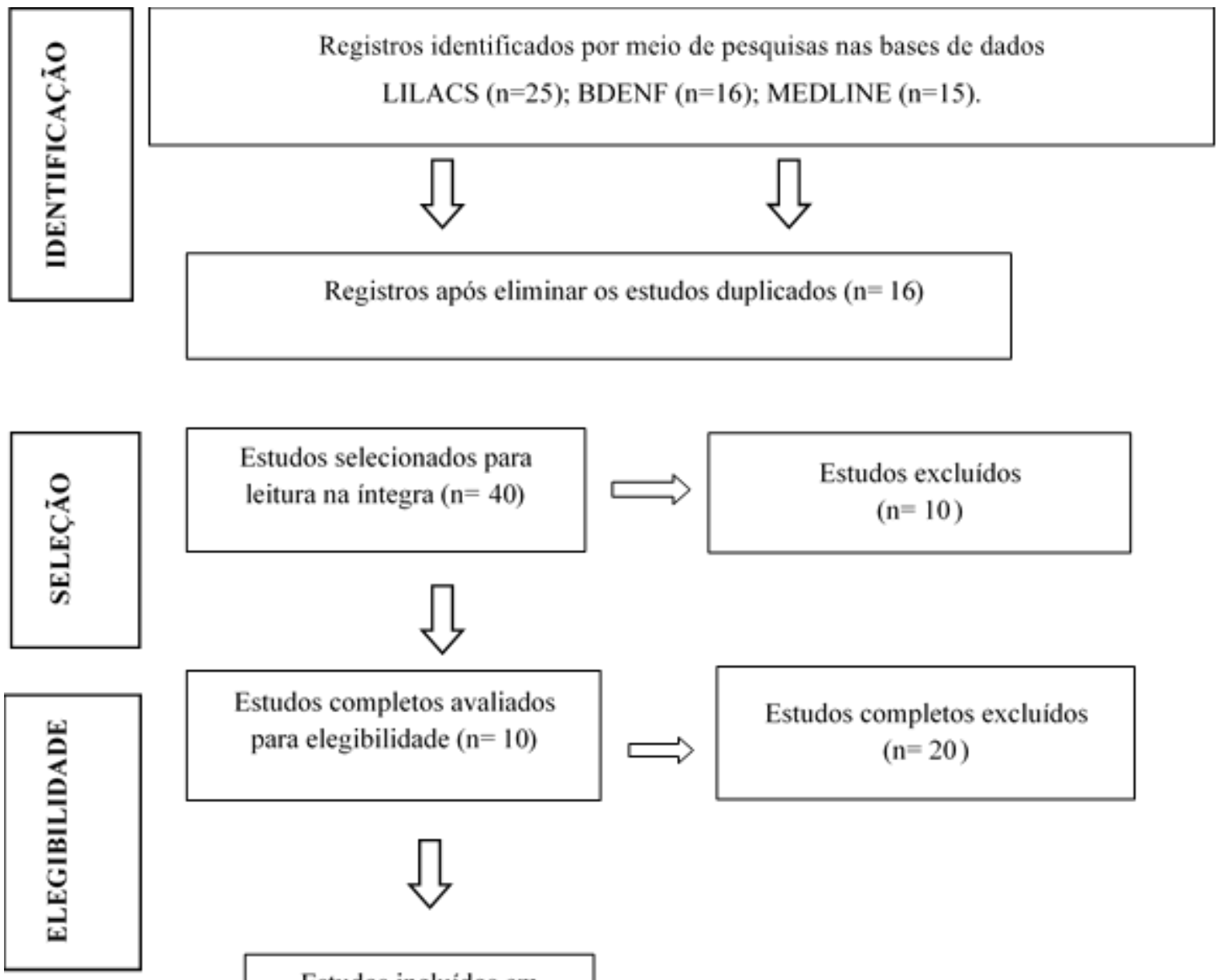

Estudos completos excluidos $(\mathrm{n}=20)$
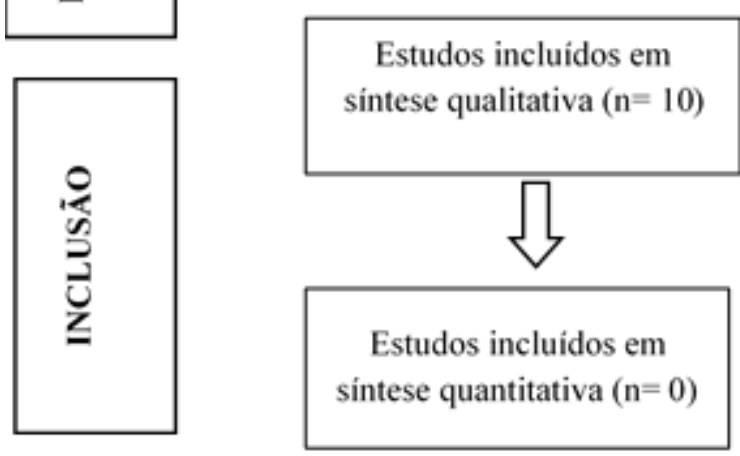

Fonte: PRISMA.

Quanto ao idioma de origem no qual a pesquisa foi publicada, nota-se predomínio no idioma Português totalizando $100 \%$ dos artigos enquanto nos idiomas Espanhol e Inglês não foram encontrados.

Quanto ao ano de publicação, os estudos selecionados foram entre os períodos dos anos 2015 a 2020 e verificou-se um maior volume nos últimos 5 cinco anos, sendo 5 artigos publicados no mesmo ano, citados a seguir: 2017 (50\%). No ano a seguir obteve-se 2 artigos: 2018 (20\%). Nos seguintes anos obteve-se 1 artigo a cada ano respectivamente: 2015 (10\%), 2016 (10\%) e 2019 (10\%). E, entre os anos 2014 e 2020, até 15 de maio deste último, não se obteve estudo que respondesse à questão da pesquisa.

Em relação ao periódico 50\% dos artigos estão publicados nas revistas dirigidas ao campo da Enfermagem, 40\% estão voltadas na área da Engenharia Sanitária Ambiental e 10\% dos artigos estão publicados nas revistas da área da Medicina. A respeito do país de origem dos estudos 100\% das publicações são oriundos da América do Sul (Brasil). 
Sobre a formação profissional dos autores dos estudos, $20 \%$ dos artigos são redigidos exclusivamente por enfermeiros; $10 \%$ redigidos exclusivamente por odontólogos; $10 \%$ redigidos exclusivamente por engenheiros sanitarista e ambiental; $10 \%$ redigido por enfermeiro e geógrafo; $10 \%$ enfermeiro e biólogo; $10 \%$ redigido pelo enfermeiro e engenheiro civil; $10 \%$ redigidos por biólogo e agrônomo; $10 \%$ redigidos por farmacêutico e engenheiro civil e $10 \%$ redigidos por tecnólogo e engenheiro químico. Conforme descrito na Tabela 1, caracterizando a redação dos artigos por cada profissional

Tabela 1 - Área de especialidades dos pesquisadores.

\begin{tabular}{lc}
\hline Área de especialidades & Quantitativo \\
\hline Enfermeiros & 16 \\
Odontologistas & 5 \\
Biólogo & 3 \\
Engenheiro sanitarista e ambiental & 4 \\
Agrônomo & 1 \\
Geógrafo & 1 \\
Tecnólogo em gestão ambiental & 1 \\
Engenheiro Químico & 1 \\
Farmacêutico & 2 \\
Engenheiro civil & 1 \\
\hline
\end{tabular}

Fonte: Dados extraídos de produções científicas recuperadas na Biblioteca Virtual em Saúde (BVS) e Base de dados MEDLINE, LILACS e BDENF.

Conforme a Tabela 1 verifica-se que a área de enfermagem foi a que mais elaborou e publicou sobre o tema Desafios do gerenciamento dos RSS, evidenciando, portanto, que o profissional enfermeiro é um dos que mais tem se comprometido no contexto dos processos de PGRS (programa de gerenciamento de resíduo).

Em relação à similaridade didática dos estudos, $80 \%$ dos estudos analisados apresentaram problemas estruturais no gerenciamento de resíduos, manifestando assim, a incapacidade de prosseguir com um gerenciamento adequado.

Quanto à qualidade da evidência observou-se a predominância dos artigos classificados como: NE $1 \mathrm{~A}$ com $60 \%$ dos artigos; NE 2C com 20\% dos artigos; NE 3B com 10\% dos artigos e NE 4 com 10\% dos artigos.

Após análise, os artigos e seus respectivos níveis de evidencias foram categorizados, conforme demonstrado na Figura 2. 
Figura 2 - Categorização dos artigos e a classificação dos níveis de evidências.

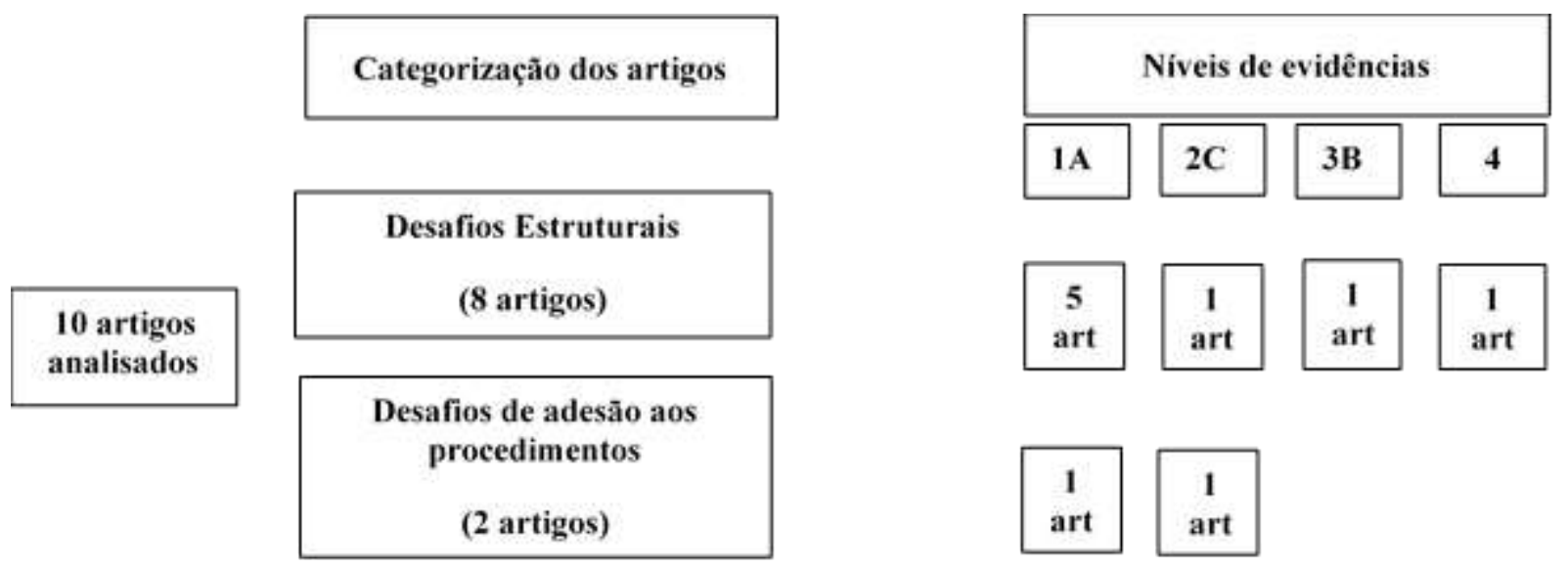

IA - Revisões sistemáticas e meta análises de ensaios clínicos comparáveis

$2 \mathrm{C}$ - Revisão sistemática homogênea de estudos de coorte

3B-Estudos de caso com grupo-controle

4- Relato de casos e série sem definição de caso controle

Fonte: Produções científicas recuperadas na Biblioteca Virtual em Saúde (BVS) e Base de dados MEDLINE, LILACS e BDENF.

A figura acima demonstra que os NE 1A e 2C exprimem um delineamento ideal, de confiabilidade e precisão em condutas para o correto gerenciamento dos RSS favorecendo a prevenção e o controle da infecção em assistência à saúde, sendo responsáveis por $80 \%$ dos estudos.

O Quadro 2 e Tabela 2 sintetizam as principais características dos estudos revisados no que se refere sobre os desafios da equipe multidisciplinar no gerenciamento de resíduos de saúde visando a prevenção e controle de infecção hospitalar.

Quadro 2 - Descrição e análise dos estudos.

\begin{tabular}{|c|c|c|c|}
\hline Ord & Autor/Ano & Título & $\begin{array}{c}\text { Desafios dos gestores e profissionais de } \\
\text { saúde }\end{array}$ \\
\hline $\mathbf{L}$ & $\begin{array}{l}\text { André, S., Veiga, } \\
\text { T., Takayanagui, A. } \\
\text { (2016) }\end{array}$ & $\begin{array}{l}\text { Geração de resíduos de serviços } \\
\text { de Saúde em hospitais de Ribeirão } \\
\text { Preto (SP), Brasil. }\end{array}$ & $\begin{array}{l}\text { - Assistência prestada inadequada, falta de } \\
\text { empenho da gerência dos RSS e da gestão } \\
\text { hospitalar, possibilitando a contaminação } \\
\text { e possíveis infecções. }\end{array}$ \\
\hline B & $\begin{array}{l}\text { CostaI, T.; FelliI, } \\
\text { V.; Sanchez, M. et } \\
\text { al (2018) }\end{array}$ & $\begin{array}{l}\text { Gerenciamento intra-hospitalar } \\
\text { dos resíduos químicos perigosos } \\
\text { manuseados pela enfermagem. }\end{array}$ & $\begin{array}{l}\text { - Gerenciamento na } \\
\text { segregação, acoquado } \\
\text { identificação e transporte interno dos } \\
\text { resíduos propiciando no ambiente } \\
\text { hospitalar infecções. }\end{array}$ \\
\hline B & $\begin{array}{l}\text { Teixeira, M.; } \\
\text { Guanilo, M.; } \\
\text { Knuth, G. et al } \\
\text { (2018). }\end{array}$ & $\begin{array}{l}\text { Avaliação da gestão dos resíduos } \\
\text { em unidades básicas de saúde de } \\
\text { um município sul-brasileiro. }\end{array}$ & $\begin{array}{l}\text { - Desconhecimento das legislações, } \\
\text { ausência de plano de Gerenciamento } \\
\text { (PGRSS), falta de material, acesso ao } \\
\text { armazenamento dos resíduos prejudicado } \\
\text { e periodicidade de coleta insuficiente, } \\
\text { alto risco de contaminação. }\end{array}$ \\
\hline $\mathbf{L}$ & $\begin{array}{l}\text { Neves B.; Lima E. } \\
\text { (2019) }\end{array}$ & $\begin{array}{l}\text { Condições da prestação dos } \\
\text { serviços ambientais de coleta e } \\
\text { destinação de resíduos de serviços } \\
\text { de saúde em unidades básicas de } \\
\text { saúde na cidade de Pelotas, RS, } \\
\text { Brasil. }\end{array}$ & $\begin{array}{l}\text { - Desconhecimento dos profissionais } \\
\text { quanto ao tratamento, separação e destino } \\
\text { dos resíduos. Ausência do plano de } \\
\text { gerenciamento (PGRSS) e uso de } \\
\text { uniformes e EPIS inadequados. } \\
\text { - Possibilidade de disseminação IRAS. }\end{array}$ \\
\hline
\end{tabular}




\begin{tabular}{|c|c|c|c|}
\hline Ord & Autor/Ano & Título & $\begin{array}{c}\text { Desafios dos gestores e profissionais de } \\
\text { saúde }\end{array}$ \\
\hline $\mathbf{L}$ & $\begin{array}{l}\text { Gomes, A., Garbin, } \\
\text { A. et al. (2017) }\end{array}$ & $\begin{array}{lr}\begin{array}{l}\text { Sustentabilidade } \\
\text { gerenciamento de }\end{array} \text { resíduos } \\
\text { odontológicos no Serviço Público }\end{array}$ & $\begin{array}{l}\text { - Desconhecimento dos profissionais sobre } \\
\text { o PGRSS. } \\
\text { - Incorreto manejo na segregação }\end{array}$ \\
\hline $\mathbf{L}$ & $\begin{array}{l}\text { Amarante, J.; Rech, } \\
\text { T.; Siegloch, A. } \\
(2017)\end{array}$ & $\begin{array}{l}\text { Avaliação do gerenciamento dos } \\
\text { resíduos de medicamentos e } \\
\text { demais resíduos de serviços } \\
\text { de saúde na Região Serrana de } \\
\text { Santa Catarina. }\end{array}$ & $\begin{array}{l}\text { - Deficiência no descarte dos resíduos de } \\
\text { medicamentos em todas as etapas do } \\
\text { gerenciamento dos RSS } \\
\text { - Inexistência do PGRSS. } \\
\text { - Rico para contaminação e propagação de } \\
\text { IRAS. }\end{array}$ \\
\hline $\mathbf{L}$ & $\begin{array}{l}\text { Siva, N.; Mosegui, } \\
\text { G. et al. (2017) }\end{array}$ & $\begin{array}{l}\text { Fuzzy Visa: um modelo de lógica } \\
\text { fuzzy para a avaliação de risco da } \\
\text { Vigilância Sanitária para inspeção } \\
\text { de resíduos de serviços de saúde. }\end{array}$ & $\begin{array}{l}\text { - Falha na adequação no período de } \\
\text { fiscalização nas etapas de coleta. } \\
\text { - Risco para contaminação. }\end{array}$ \\
\hline B & $\begin{array}{l}\text { Dias, G.; Sarturi F.; } \\
\text { Campongara S.et } \\
\text { al. (2017). }\end{array}$ & $\begin{array}{l}\text { Análise da taxa de geração de } \\
\text { resíduos de serviços de saúde em } \\
\text { um hospital universitário. }\end{array}$ & - Falta de proatividade do Gestor \\
\hline $\mathbf{M}$ & $\begin{array}{l}\text { Camargo, A. \& } \\
\text { Melo, I. (2017) }\end{array}$ & $\begin{array}{l}\text { A percepção profissional sobre o } \\
\text { gerenciamento dos resíduos de } \\
\text { serviços de saúde em unidades } \\
\text { básicas e ambulatórios de saúde } \\
\text { em um município da Região } \\
\text { Metropolitana de } \\
\text { Sorocaba, SP, Brasil. }\end{array}$ & $\begin{array}{l}\text { - Falta de estrutura física e recursos } \\
\text { humanos. } \\
\text { - Conhecimento inadequado sobre as } \\
\text { normas dispostas no PGRSS } \\
\text { - Conhecimento insuficiente no GRSS. } \\
\text { - Deficiência da Educação permanente, } \\
\text { - permitindo uma possível IRAS. }\end{array}$ \\
\hline $\mathbf{L}$ & $\begin{array}{l}\text { Maders, G. \& } \\
\text { Cunha, H. (2015) }\end{array}$ & 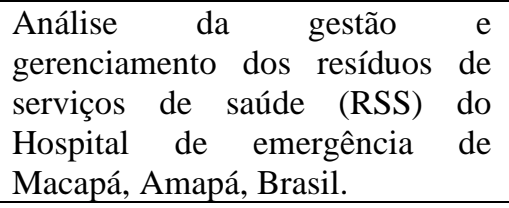 & $\begin{array}{l}\text { - Falta da apropriação do PGRSS, } \\
\text { - Ausência de infraestrutura adequada. }\end{array}$ \\
\hline
\end{tabular}

Legenda: M: MEDLINE; L: LILACS; B: BDENF.

Fonte: Produções científicas recuperadas na Biblioteca Virtual em Saúde (BVS) e Base de dados MEDLINE, LILACS e BDENF. 
Tabela 2 - Principais características dos artigos revisados.

\begin{tabular}{|c|c|c|}
\hline Categorias & Variantes & Quantidade \\
\hline $\begin{array}{l}\text { País de } \\
\text { publicação/Continente }\end{array}$ & América do Sul (Brasil) & 10 \\
\hline \multirow[t]{6}{*}{ Periódico/foco e escopo } & Eng Sanit Ambient & 4 \\
\hline & Revista de enferemagem UERJ & 1 \\
\hline & R.Pesq.Cuid.Fundam. On line & 2 \\
\hline & Rev. Cuba. Estomatol & 1 \\
\hline & Physis Revista de Saúde Coletiva & 1 \\
\hline & Rev. Mundo Saúde & 1 \\
\hline \multirow{6}{*}{$\begin{array}{l}\text { Área de conhecimento } \\
\text { do primeiro autor }\end{array}$} & Enfermagem & 5 \\
\hline & Técnica química & 1 \\
\hline & Bióloga & 1 \\
\hline & Odontologista & 1 \\
\hline & Engenheiro & 1 \\
\hline & Advogado ambiental & 1 \\
\hline $\begin{array}{l}\text { Idioma de origem da } \\
\text { publicação }\end{array}$ & Português & 10 \\
\hline \multirow[t]{4}{*}{ Delineamento } & Descritivo exploratório quantitativa & 6 \\
\hline & Transversal (seccional) & 2 \\
\hline & Estudo de caso & 1 \\
\hline & Relato de experiência & 1 \\
\hline \multirow[t]{4}{*}{ Níveis de evidência } & 1A- Revisões sistemáticas e meta análises de ensaios clínicos comparáveis & 6 \\
\hline & 2C- Revisão sistemática homogênea de estudos de coorte & 2 \\
\hline & 3B - Estudos de caso com grupo-controle & 1 \\
\hline & 4- Relatos de casos e série sem definição de caso controle & 1 \\
\hline \multirow{4}{*}{$\begin{array}{l}\text { Resposta à questão de } \\
\text { pesquisa }\end{array}$} & Ausência do PGRSS & 3 \\
\hline & Incorreto manejo na segregação, utilização inadequada de EPI & 2 \\
\hline & Ausência da Educação Permanente & 2 \\
\hline & Ausência dedicação da gerência & 3 \\
\hline
\end{tabular}

Fonte: Dados extraídos de produções científicas recuperadas na Biblioteca Virtual em Saúde (BVS), Base de dados MEDLINE, LILACS e BDENF.

\section{Discussão}

Através da investigação identificou-se conflitos socioambientais, envolvendo questões importantes dos RSS, tendo como consequência infecção relacionada à assistência a saúde (IRAS) como um grande risco para a saúde pública. Então, expõe-se a necessidade de discussão, elaboração e implementação de ações de gerenciamento de resíduos de saúde (GRSS), que visem o controle e prevenção de infecção relacionada à assistência a saúde. Desta forma, as pesquisas vêm evidenciando diversos desafios enfrentados pelos gestores e também pela equipe multidisciplinar ao gerenciar os resíduos de serviço de saúde.

Dentre os $80 \%$ dos artigos observou-se que a administração, por algumas vezes acontece de forma inadequada e insatisfatória. Para cumprirem-se as determinações legais e alcançar um gerenciamento de resíduos de sérvio de saúde (GRSS) adequado, revela-se uma série de desconformidades que necessitam de aprimoramento no atendimento às suas especificações, desde o ato de gerenciar as etapas nos processos tais como: segregação, acondicionamento, identificação e transporte internamente dos resíduos.

Detectou-se a necessidade da aplicabilidade de um plano que levem os profissionais a atuarem de forma técnica adequada, tendo em vista adquirir efeitos positivos, os quais os estimulem a repensar as práticas cotidianas. Contribuindo na 
qualidade dos serviços de saúde, gerando um melhor controle e execução do manejo adequado dos RSS, em especial da etapa da segregação e manejo (Freitas \& Silva, 2012).

Observou-se no estudo que o maior desafio da equipe multidisciplinar no gerenciamento de resíduos de saúde está associado à falta de capacitação profissional, assim como o desconhecimento das legislações vigentes que tratam sobre os resíduos de serviços de saúde, entre outros. Além disto, verificou-se que em $20 \%$ dos resultados, os artigos analisados demonstraram o uso inadequado do EPI pelos profissionais e o manejo incorreto das etapas de gerenciamento.

Mediante a isto, elaborou-se duas categorias: Desafios estruturais e Desafios de adesão dos profissionais aos procedimentos.

\subsection{Desafios estruturais}

A prevalência da escassez estrutural e administrativa foi observada em $80 \%$ dos estudos, um indicativo de péssimas condições que envolve o processo de trabalho e gerenciamento de resíduos de saúde (Uehara, 2019; $\quad$ Costa et al., 2018; Teixeira et al., 2018; Amarante et al., 2017; Silva et al., 2017; Dias et al., 2017; Camargo \& Melo, 2017; Maders \& Cunha, 2015).

Evidenciou-se a falta de capacitação dos funcionários envolvidos no manejo dos RSS. Tendo em vista, que seus manuseios devem ser feitos com critérios, de forma cuidadosa e responsável garantindo a segurança e reduzindo os riscos e exposição à saúde humana (Uehara, 2019; Teixeira et al., 2018; Amarante et al., 2017; Camargo \& Melo, 2017; Neves \& Lima, 2019; Gomes et al., 2017).

Em outro estudo que avaliou o manejo do RSS, a falta de capacitação e o consequente desconhecimento sobre RSS e PGRSS ficaram evidenciados, corroborando, portanto com a temática deste estudo (Bento et al., 2017).

Esses achados estão em consonância com uma pesquisa desenvolvida que descreve os motivos para um gerenciamento inadequado dos RSS: ausência de preocupação referente aos resíduos perigosos dos RSS, treinamento inadequado para o gerenciamento de resíduos, falta de gerenciamento de resíduos e disposição, recursos financeiros e humanos insuficientes e a não devida prioridade dada para a temática (Garcia \& Ramos, 2004).

Contudo, observou-se a ausência de plano de gerenciamento, ocasionando falhas na segregação, acondicionamento, identificação e transporte interno dos resíduos. Irregularidades como essas, podem ocasionar vastos prejuízos ao meio ambiente e aos trabalhadores (Teixeira et al., 2018; Neves \& Lima, 2019; Amarante et al., 2017).

Além disso, uma questão constatada no estudo é que os profissionais da assistência põem todos e quaisquer procedimentos vinculados à assistência direta ao paciente como primordial, ausentando-se das questões relacionadas ao PGRSS, sendo esse um programa sem destaque para os profissionais assistencialistas, colocando-se como potenciais risco de propagação de IRAS (Camargo \& Melo, 2017).

Tendo em vista que o processo de gerenciamento de RSS começa com um PGRSS, logo, um planejamento adequado dos resíduos, aliado a capacitação dos profissionais, podem contribuir significativamente na redução da ocorrência de acidentes de trabalho, especialmente aqueles provocados por perfurocortantes.

Além dos trabalhadores dos serviços de saúde, também os trabalhadores das companhias de limpeza manuseiam os resíduos de serviços de saúde, e são expostos aos riscos inerentes quando esses resíduos são mal gerenciados, assim como os graves danos causados ao meio ambiente com a inadequada eliminação desses sedimentos. Torna-se relevante neste sentido, que gestores, coordenadores e toda equipe multidisciplinar das unidades de saúde, conheçam a legislação e a importância de um adequado PGRSS, a fim de diminuir os danos ambientais causados pela prática (Garcia \& Ramos, 2004). 
Concomitantemente com outros estudos que enfatizam que a falta do PGRSS compromete a gestão, resultando em maior risco, tanto para os profissionais atuantes nos serviços de saúde, quanto para os profissionais externos que coletam os resíduos (Bento et al., 2017; Rizzon et al., 2015).

A utilização de um PGRSS é de muita relevância, uma vez que enfatiza a conscientização dos profissionais e de todos envolvidos quanto à responsabilidade de sua função no cuidado dos RSS, possibilitando uma segregação de resíduos infectados e não infectados, que potencializará a resolução de uma parte do problema do gerenciamento inadequado (Rizzon et al., 2015).

Aliado a isto, os pontos críticos podem ser sanados, pois no plano as normatizações são abordadas de formas diferentes em relação a cada processo com intenção de realizar um gerenciamento de resíduos adequado. Visto que, este plano organiza, documenta, descreve e orienta as práticas a serem adotadas por todos os profissionais atuantes (Rizzon et al., 2015).

Outro desafio exposto frequentemente no estudo foi à coleta, sendo classificada como desorganizada, acessos dificultosos para todos os profissionais que manuseiam RSS nas unidades produtora. Pode -se verificar que em $40 \%$ das pesquisas, informam que a periodicidade é insuficiente, considerando o fluxo de demanda (Teixeira et al., 2018; Amarante et al., 2017; Silva et al., 2017; Neves \& Lima, 2019).

Está problemática também foi destacada no estudo realizado em uma Instituição Pública de Saúde e Ensino Superior da região sul do país, e acredita que a implantação de uma política na instituição relacionada ao gerenciamento de resíduos favorece para ações de manejo adequado (Corrêa et al., 2007).

Relembrando que é dever das unidades geradoras de RSS, que a administração de seus resíduos desde a produção até o destino final, seja realizada conforme previsto na RDC 222/18 (Ministério da Saúde, 2018).

Outro achado no estudo foi a falta de proatividade dos gestores para solucionar problemas como: construção do depósito de armazenamento externo dos RSS, rampas para diminuir o trajeto interno no transporte, estruturas para manuseio específico tufo isso considerando o tratamento interno obrigatório. (Camargo \& Melo, 2017; Maders \& Cunha, 2015).

Refletindo-se na fragilidade e desafio do gerenciamento de resíduos de saúde na prevenção e controle da infecção hospitalar da equipe multidisciplinar, encontra-se a falta de materiais para o correto manejo e a ausência de treinamento em relação ao tratamento e gerenciamento dos RSS.

Em outro estudo é enfatizado que 54,5\% dos gestores demonstraram desconhecimento da existência do Plano de Gerenciamento de Resíduos de Serviço de Saúde (PGRSS) da instituição. Evidenciando-se déficit no conhecimento dos trabalhadores, profissionais e gestores acerca do PGRSS, bem como práticas inadequadas no manejo dos RSS. Ao mesmo tempo $81,8 \%$ dos gestores desconhecem a legislação que regulamenta o plano bem como a existência de licença ambiental para o funcionamento da Instituição, o mapa de risco e o destino final dos RSS produzidos no serviço (Mendes et al., 2015). Sendo assim, justifica-se a falta de proatividade e iniciativa dos gestores. Sem conhecimento não se organiza, não se projeta e não se gerencia.

\subsection{Desafios de adesão dos profissionais aos procedimentos}

A falta do uso de uniformes adequados e equipamento de proteção individual (EPIs) pelos profissionais foram observados em $20 \%$ dos estudos, realidade esta que é alarmante, visto que se tem como objetivo do EPI a proteção individual dos funcionários e a preservação da saúde (Neves \& Lima, 2019; Gomes et al., 2017). De acordo com a NR-6 é de responsabilidade do empregador o fornecimento aos empregados, o EPI adequado ao risco, com estado de conservação perfeito e funcionamento correto (Ministério da Saúde, 2018).

Desta forma, o uso inadequado dos EPIs por parte dos profissionais gera uma necessidade de reeducação técnica, quanto a seu nível de importância e utilização. Ainda que, os profissionais usem os equipamentos de proteção individual durante o manuseio dos resíduos, foi observado também a falta de compreensão e conhecimento de todos, somado ao 
desconhecimento por parte dos executores dos processos de coletas específicos. Sendo assim, colaboram para o aumento de riscos iminentes e IRAs (infecção relacionada à assistência à saúde) (Neves \& Lima, 2019; Gomes et al., 2017).

Nesse sentido, observa-se a necessidade da aplicação de um programa de educação permanente previsto pela Portaria no 1996/07/GM/MS (Ministério da Saúde, 2018; Conselho Nacional do Meio Ambiente, 2005), que tem como proposta a qualificação e transformação das práticas de saúde e, desta forma, realizar cuidado de forma integral a população. A Educação permanente em saúde (EPS) apresenta ações com propósito de implementar práticas reflexivas, éticas e humanísticas com enfoque na equipe e no ambiente do trabalho (Massaroli \& Saupe, 2008; Porto et al., 2019).

Corroborando com o estudo Coswosk et al. (2017) que elaboraram módulos de cursos de acordo com a formação técnica do profissional sobre o Gerenciamento dos RSS, favoreceu assim o entendimento da importância de cada profissional no processo de implementação do PGRSS. Abordando as seguintes temáticas: Apresentação e importância do PGRSS, implementação do conceito de ecoeficiência nas rotinas de trabalho, noções gerais sobre o ciclo da vida dos materiais; a importância dos POP'; conhecimento da legislação ambiental e vigilância sanitária relativas aos RSS; definições, tipos e classificação dos resíduos e seu potencial de risco; orientações sobre biossegurança (biológica química e radiológica); sistema de gerenciamento adotado internamente no estabelecimento; formas de reduzir a geração de resíduos e reutilização de materiais; conhecimento das responsabilidades e de tarefas; orientações sobre o uso de Equipamentos de Proteção Individual EPI' e Coletiva - EPC' específicos de cada atividade, bem como sobre a necessidade de mantê-los em perfeita higiene e estado de conservação; orientações sobre higiene pessoal e dos ambientes; conhecimento sobre a utilização dos veículos de coleta; técnicas de higienização com objetivo auxiliar na redução dos índices de infecção hospitalar.

Observou -se no fim do curso que, a introdução de conceitos relacionados ao manejo adequado de resíduos levou maior abrangência na compreensão da temática e favoreceu para mudanças de condutas profissionais e pessoais (Coswosk et al., 2017).

Desta forma, é notável a importância da educação permanente entre os profissionais de saúde, impedindo a estagnação profissional e pessoal desses elementos, gerando um processo influente no desenvolvimento crítico e na percepção de que a busca de ensino é instrumento para aprendizagem. A educação reforça para construção do conhecimento e a sua aquisição permite a constituição de sujeitos sociais capazes de instituir sua identidade, desenvolver sua racionalidade e assim tomar decisões na vida social, política e econômica. O conhecimento dá ao ser humano a oportunidade de desenvolver habilidades e competências que são exigidas para usufruir do conjunto de bens e serviços disponíveis na sociedade e, enfim, para comunicação e aprendizado (Coswosk et al., 2017).

\section{Conclusão}

Com o estudo, certificou-se que os desafios da equipe multidisciplinar no gerenciamento de resíduos são inúmeros. No entanto, apontamos questões na estrutura física e humana, assim como, na administração de RSS em particular com a falta de adequação do PGRSS.

A ausência de infraestrutura correta e a inadequada preparação do manejo, relacionado com a falta de capacitação profissional e também o desconhecimento a respeito das legislações atuais que administram os resíduos de serviços de saúde (RSS), permitem que PGRS sejam insuficientes ou ausentes.

É correto afirmar que o gerenciamento de RSS apropriado para a clínica trabalhada, contribui na redução da infecção hospitalar, minimizando os riscos aos profissionais envolvidos no manejo e também para os pacientes assistidos, assim como para o ambiente.

Vale ressaltar que a educação permanente e continuada voltada para a capacitação dos funcionários contribui no controle da infecção, garantindo a segurança e reduzindo as ameaças de exposição à saúde humana. 
Devido ao Gerenciamento de resíduos de saúde ser essencial, os riscos impostos à saúde pública e ambiental, por falta de gerenciamento de resíduos de saúde, são vastos, podendo potencializar o aumento da taxa de infecções relacionadas à assistência à saúde. Este artigo permite que futuros pesquisadores utilizem este estudo para identificar a importância de PGRS, assim como, os desafios que são encontrados para executá-los. Recomenda-se para futuros estudos a abordagem desse tema, a fim de identificar mudanças nessa prática.

\section{Referências}

Agência Nacional de Vigilância Sanitária [ANVISA]. (2018). Resolução da Diretoria Colegiada RDC nº 222, de 28 de março de 2018. Regulamenta as Boas Práticas de Gerenciamento dos Resíduos de Serviços de Saúde e dá outras providências. http://portal.anvisa.gov.br/documents/33852/271855/RD C+222+de+Mar\%C3\%A7o+de+2018+COMENTADA/edd85795-17a2-4e1e-99ac-df6bad1e00ce

Alves, D. C. I., \& Lacerda R. A. (2015). Avaliação de programas de controle de infecção relacionada a assistência à saúde. Revista Escola de Enfermagem, 49(Esp), 65-73. http://www.scielo.br/scielo.php?script=sci_arttext\&pid=S0080-62342015000700065.

Amarante, J.A.S., Rech, T. D., \& Siegloch, A. E. (2017). Avaliação do gerenciamento dos resíduos de medicamentos e demais resíduos de serviços de saúde na Região Serrana de Santa Catarina. Revista Engenharia Sanitária Ambiental, 22(2), 317-326. https://www.scielo.br/pdf/esa/v22n2/1809-4457-esas1413_41522016150080.pdf

Bento, D. G., Costa, R., Luz, J. H., \& Klock, P. (2017). O gerenciamento de resíduos de serviço de saúde sob ótica dos profissionais de saúde. Texto e contexto Enfermagem, 26(1):e6680015. https://www.scielo.br/pdf/tce/v26n1/pt_1980-265X-tce-26-01-e6680015.pdf

Camargo, A. R., \& Melo, I. M. N. (2017). A percepção profissional sobre o gerenciamento dos resíduos de serviços de saúde em unidades básicas e ambulatórios de saúde em um município da Região Metropolitana de Sorocaba, SP, Brasil. Mundo Saúde, 41(4), 633-643. https://pesquisa.bvsalud.org/portal/resource/pt/mis-40083

Conselho Nacional do Meio Ambiente [CONAMA]. (2005). Resolução $n^{o}$ 358, de 29 de abril de 2005. Dispõe sobre o tratamento e a disposição final dos resíduos dos serviços de saúde e dá outras providências. Conama.

Corrêa, L. B., Lunardi, V. L., \& Conto, S. M. (2007). O processo de formação em saúde: o saber resíduos de saúde em vivências práticas. Revista Brasileira de Enfermagem, 60(1), 21-35. https://www.scielo.br/pdf/reben/v60n1/a04v60n1.pdf

Costa, T. F., Felli, V. E. A., Sanchez, M. C. O., Ferreira, S. C. M., Silvino, Z. R \& Souza, D. F. (2018). Gerenciamento intra-hospitalar dos resíduos químicos perigosos manuseados pela enfermagem. Revista Enfermagem UERJ, 26, e19376. publicacoes.uerj.br/index.php/enfermagemuerj/article/view/19376

Coswosk, E. D., Rosa, C. G. S., Caldeira, A. B., Silva, N. C. R., \& Rocha, J. M. (2018). Educação continuada para o profissional de saúde no gerenciamento de resíduos de Saúde. Revista Brasileira de Análises Clínicas, 50(3),288-296. http://www.rbac.org.br/wp-content/uploads/2019/01/RBAC-vol-50-3-2018-ref645-final.pdf

Dias, G. L., Sarturi, F., Camponogara, S., Lima, S. B. S., Lopes, L. F. D., \& Trevisan, C. M (2017). Análise da taxa de geração de resíduos de serviços de saúde em um hospital universitário. Journal of Research: fundam. 9(1), 92-98. http://www.seer.unirio.br/index.php/cuidadofundamental/article/view/4889/pdf_1

Freitas, I. M., \& Silva, M. A. (2012). A importância do gerenciamento de resíduos do serviço de saúde na proteção do meio ambiente. Revista Estudos, 39(4),493-505. http://www.seer.ufu.br/index.php/hygeia/article/viewFile

Garcia, L. P., Ramos, B. G. Z. (2004). Gerenciamento dos resíduos de serviços de saúde: uma questão de biossegurança. Cadernos de Saúde Pública,20(3),744-752. https://www.scielo.br/scielo.php?pid=S0102311X2004000300011\&script=sci_abstract\&tlng=pt

Giarola, L. B., Baratieri, T., Costa, A. M., Bendendo, J., Marcon S. S., \& Waidman, M. A. P. (2010). A infecção hospitalar na perspectiva dos profissionais de enfermagem: um estudo bibliográfico. Cogitare Enferm UEM, 17(1),151-157. https://revistas.ufpr.br/cogitare/article/view/26390

Gomes, A. M. P., Garbin, A. J. I., Arcieri, R. M., Rovida, T. A. S., \& Garbin, C. A. S.(2017). Sustentabilidade ambiental: gerenciamento de resíduos odontológicos no Serviço. Revista Cubana de Estomatología, 54(2), 1-11. https://pesquisa.bvsalud.org/portal/resource/pt/biblio-901031

Maders, G. R., \& Cunha, H. F. A. (2015). Análise da gestão e gerenciamento dos resíduos de serviços de saúde (RSS) do Hospital de Emergência de Macapá, Amapá, Brasil. Revista Engenharia Sanitária Ambiental, 20(3), 379-388. https://www.scielo.br/pdf/esa/v20n3/1413-4152-esa-20-03-00379.pdf

Massaroli, A., \& Saupe, R. (2008). Distinção conceitual: educação permanente e educação continuada no processo de trabalho em saúde. PIBIC. http://www1.saude.rs.gov.br/dados/1311947098405educa\%E7\%E3o\%20continuada\%20 e\%20permanente.pdf

Mendes, W., Figueiredo, M., Salmito, M., Araújo, E., Araújo, T., \& Andrade, E. (2015). Knowledge and practice of workers, professionals and managers on waste of health services. Revista de Pesquisa: Cuidado é Fundamental Online, 7(4), 3216-3226. http://www.seer.unirio.br/index.php/cuidadofundamental/article/view/3684

Ministério da Saúde. (1998). Portaria nº 2616/MS/GM, de 12 de maio de 1998. Programa de Controle de Infecção Hospitalar. Ministério da Saúde.

Ministério da Saúde. (2006). Gerenciamento dos resíduos de serviços de saúde. Agência Nacional de Vigilância Sanitária. http://www.anvisa.gov.br/servicosaude/manuais/manual_gerenciamento_residuos.pdf. 
Ministério da Saúde. (2018). Resolução da Diretoria Colegiada - RDC n $n^{o} 222$, de 28 de março de 2018. Regulamenta as boas práticas de Gerenciamento dos Resíduos de serviços de saúde e dá outras providências. Ministério da Saúde.

Ministério do Trabalho e Emprego. (2017). Portaria $n^{\circ}$ 870, de 06 de julho de 2017. Aprova a Norma Regulamentadora ${ }^{\circ} 6$ (EPI - Equipamento de Proteção Individual). Ministério do Trabalho e Emprego.

Neves, B. C., \& Lima, E. P. P. (2019). Condições da prestação dos serviços ambientais de coleta e destinação de resíduos de serviços de saúde em unidades básicas de saúde na cidade de Pelotas, RS, Brasil. Revista Engenharia Sanitária Ambiental, 24(1),61-69. https://www.scielo.br/scielo.php?script=sci_arttext\&pid=S1413-41522019000100061\&tlng=pt

Pedrosa, K. K., Oliveira, I. C. M., Feijão, A. R, \& Machado, R. C. (2015). Enfermagem baseada em evidência: caracterização dos estudos no Brasil. Cogitare Enfermagem, 20(4):733-774.

Pereira, A. S., Shitsuka, D. M., Parreira, F. J, \& Shitsuka, R. (2018). Metodologia da pesquisa científica. Editora UFSM. https://repositorio.ufsm.br/bitstream/handle/1/15824/Lic_Computacao_Metodologia-Pesquisa-Cientifica.pdf?sequence=1

Porto, M. A. O. P., Sanchez, M. C. O., Xavier, M. L., Chrizostimo, M. M., Brandão, E. S., \& Rosa, M. V.(2019). Educação Permanente em saúde: Estratégia de prevenção e controle de infecção hospitalar. Revista Nurs, 22(258), 3362-3370. https://pesquisa.bvsalud.org/portal/resource/pt/biblio-1053418

Rizzon, F., Nodari, C. H., \& Reis, Z. C. (2015). Desafio no gerenciamento em serviços públicos de saúde. Revista de Gestão em Sistemas de Saúde, 4(1), 4054. http://www.revistargss.org.br/ojs/index.php/rgss/article/view/141

Santos, C. M. C., Pimenta, C. A. M., \& Nobre, M. R. C. (2007). A estratégia PICO para a construção da pergunta de pesquisa e busca de evidências. Revista Latino-Americana de Enfermagem,15(3), $\quad$ 508-11. https://www.scielo.br/scielo.php?script=sci_abstract\&pid=S0104$11692007000300023 \& \operatorname{lng}=$ en\&nrm=iso\&tlng=en

Sasso, M. K. D., Pereira, S. R. C. C., \& Maria G. C. (2008). Revisão integrativa: método de pesquisa para a incorporação de evidências na saúde e na enfermagem. Revista Texto Contexto - Enfermagem, 17(4), 758-764. http://dx.doi.org/10.1590/S0104-07072008000400018.

Silva, N. F. C., Vianna, C. M. M., Oliveira, F. S. G., Mosegui, G. B. G., \& Rodrigues, M. P. S. (2017). Fuzzy Visa: um modelo de lógica fuzzy para a avaliação de risco da Vigilância Sanitária para inspeção de resíduos de serviços de saúde. Physis, 27(1), 127-46. https://www.scielo.br/scielo.php?script=sci_arttext\&pid=S0103-73312017000100127

Silva, R. M. (2019). Gestão hospitalar e meio ambiente: uma reflexão sobre os processos de gerenciamento de efluentes em lavanderias hospitalares. https://www.monografias.ufop.br/bitstream/35400000/1951/1/monografia_Gest\%C3\%A3o HospitalarAmbiente.pdf.

Sodré, M. S., \& Lemos, C. F. (2017). O cenário do gerenciamento dos resíduos de serviços de saúde no Brasil. Fórum Internacional de Resíduos Sólidos.

Tavares, S. M. T., Dias, S. M., \& Carvalho, R. (2010). Revisão integrativa: o que é e como fazer. Revista Einstein, 8(1), 102-106. http://www.scielo.br/scielo.php?script=sci_arttext\&pid=S167945082010000100102\&lng=en. https://doi.org/10.1590/s 1679-45082010rw1134.

Teixeira, M. V., Echevarría Guanilo, M. E., Knuth F. G. \& Ceolin, T. (2018). Avaliação da gestão dos resíduos em Unidades Básicas de Saúde em um município Sul- Brasileiro. Journal of Research: http://www.seer.unirio.br/index.php/cuidadofundamental/article/viewFile/6237/pdf_1

Uehara, S. C. S. A. (2019). Gerenciamento de resíduos de serviços de saúde em hospitais de Ribeirão Preto (SP), Brasil. Revista de Engenharia Sanitária Ambiental, 24(1), 121-130. https://www.scielo.br/scielo.php?script=sci_arttext\&pid=S1413-41522019000100121

World Health Organization. (2018). Health-care waste. https://www.who.int/news-room/fact-sheets/detail/health-care-waste 